RAPHISA.

Revista de Antropología y Filosofía de lo Sagrado Review of Anthropology and Philosophy of the Sacrum ISSN: 2530-1233 $\mathrm{N}^{\mathrm{o}}$ 1, enero-junio (2017) pp.: 137-146

\title{
NOTAS
}

\section{CHARLES TAYLOR Y LA RELIGIÓN EN LA ACTUALIDAD}

\author{
Manuel Sánchez Matito \\ Universidad de Sevilla
}

\section{La religión en un mundo secular}

Pero la voluntad de Dios puede todavía estar presente para nosotros en el designio de las cosas, en el cosmos, el Estado y la vida personal. Dios puede parecer la fuente ineludible para nuestro poder de impartir orden a nuestras vidas, tanto individual como socialmente. ${ }^{1}$

Estas palabras aparecen en los momentos finales de "On social imaginary", conferencia pronunciada por el filósofo canadiense Charles Taylor en Edimburgo en 1999. En ella hace referencia al proceso de secularización describiéndolo cómo una de las rasgos definitorios de la Modernidad. Con el término secularización quiere destacar cómo en el espacio público actual las discusiones no apelan a un fundamento último situado en un tiempo sagrado o fundacional; por el contrario, las discusiones y los acuerdos políticos se desarrollan en el presente, entre los miembros de esa sociedad. Por tanto, secular no significa, para Taylor, ausencia de religión; lo secular -siguiendo su sentido etimológico: "en el siglo"- define aquello que ocurre en el tiempo profano, en el tiempo actual.

Aunque Taylor desarrolla este tema en sus últimos escritos, el problema de la secularización ya aparece en su obra más conocida, Fuentes del yo. En esta obra relaciona Taylor la secularización con dos elementos:

[1] Ch. TAYLOR, "On social imaginary" en <www.nyu.edu/classes/calhoun/Theory/Taylor-onsi.htm>. La conferencia también se ha publicado en la revista Public Culture (cfr.: Public Culture, Volume 14, Number 1, Winter 2002). Por otra parte, esta conferencia representa el núcleo de la obra Modern Social Imaginaries, Duke University Press, Durham, Londres, 2004, que ha sido publicada en español: Ch. TAYLoR, Imaginarios sociales modernos, Paidós, Barcelona, 2006. 
el retroceso de la creencia en Dios y la decadencia de la práctica religiosa. El más significativo es el primero ya que, hasta finales del siglo XVIII, el ateísmo representaba un fenómeno muy extraño. Ahora bien, los cambios producidos en las culturas occidentales no deben explicarse, en su opinión, como consecuencia directa de transformaciones sociales (una nueva forma de vida ligada al desarrollo industrial) o como producto de un mayor desarrollo del género humano (un avance en la racionalidad); el motivo más importante es la aparición de nuevas fuentes morales que ya se han hecho disponibles en esta época. ${ }^{2}$ La lenta configuración de nuevas fuentes morales (el naturalismo científico y el expresivismo romántico) que se han ido fraguando a lo largo de la Modernidad han permitido que en la cultura occidental se resquebraje el dominio omnipotente del patrón teísta y aparezcan otras opciones morales que acentúan el valor de la racionalidad como una facultad autónoma o que destacan la importancia de los sentimientos.

En una obra más reciente, A secular Age, parte de la misma idea para recordarnos que la secularización de las culturas occidentales es un hecho que se constata al compararlas con las no occidentales o con las desarrolladas en una época anterior. A partir de este hecho, Taylor explica que la secularización puede comprenderse en tres sentidos diferentes: el "vaciado" de Dios que se ha producido en el Estado y en las diferentes esferas sociales; la falta de intensidad en las creencias y en las prácticas, y, por último, el hecho de que Dios aparece en el presente como una alternativa más entre otras y no como una realidad incuestionable y única. ${ }^{3} \mathrm{~A}$ lo largo de esta obra, Taylor empleará el término secularización siguiendo el tercer significado, mostrando la compatibilidad entre la religión y otras fuentes morales diferentes. De este modo, nos encontramos ante la tesis que frecuentemente analiza y defiende el filósofo canadiense en los últimos años: la Modernidad es secular sin dejar de ser religiosa.

\section{Individualismo y objetividad en la religión del presente}

En el párrafo que hemos destacado al comienzo de este estudio se muestra de qué modo la religión debe seguir siendo importante en un mundo secular. Dios aparece como un valor, como un bien necesario tanto desde el punto de vista individual como desde el punto de vista social. En la Modernidad la religión no desaparece, sino que pasará a ocupar un lugar diferente. Es evidente que la religión personal sigue presente en las

[2] Ch. TAYlor, Fuentes del yo, Paidós, Barcelona, 1996, op. cit., pp. 328 y ss.

[3] Ch. TAylor, A secular age, The Belknapp of Harvard University Press, Cambridge, Massachussets, y Londres, 2007, pp. 1-22. 
sociedades contemporáneas, pero, además, sigue desempeñando un papel importante en la vida pública, tal y como refleja, según nos dice Taylor, el caso norteamericano.

En una entrevista realizada por Bruce Ellis Benson "What it means to be secular?", ${ }^{4}$ Taylor define la vida secular como aquella que se desarrolla en un mundo en el que Dios ya no es inevitable, lo cual no significa vivir en un mundo en el que Dios haya sido expulsado. Cree que todavía hay un lugar para la religión y para Dios en este mundo secular, tanto en el aspecto personal - a través de la devoción - como en el mundo social, donde la ausencia de un orden sagrado puede sustituirse por un fuerte sentido de Dios en nuestra identidad política.

En esta misma entrevista Taylor destaca la pluralidad de nuestras fuentes morales (el naturalismo de la racionalidad desvinculada, el expresivismo romántico o la tradición teísta) y la conflictividad entre las mismas como una de las notas características del desarrollo moderno. Taylor no cree que la religión por su propia esencia anule esta conflictividad. Sí podría hacerlo alguna creencia en particular o alguna ideología; pero esto habría que evitarlo, pues cualquier intento de homogeneización o unanimidad puede conducir a un terrible desenlace.

Hay perspectivas rivales, mutuamente contradictorias, pero ninguna es capaz de derribar a todas las demás. Esto es lo que significa vivir en una civilización secular (...) Y cualquier intento por imponer cierta unanimidad, ya sea desde un modelo ateísta o teísta, marchará hacia un terrible fin... ${ }^{5}$

Por tanto, la religión puede seguir desempeñando un importante papel en una Modernidad caracterizada por el pluralismo y la secularidad. Ésta es una de las notas distintivas de la religiosidad en la actualidad. Pero hay otro aspecto que Taylor desea destacar constantemente: siendo evidente el proceso de individualización e interiorización que se ha desarrollado a lo largo de la Modernidad, afectando tanto al ámbito de la moral como al fenómeno religioso, no es posible explicar la importancia actual de la religión aludiendo únicamente a este fenómeno. Aunque la religión en la Modernidad se ha visto afectada por un importante giro hacia el interior, continúa adoptando una dimensión objetiva y pública.

Esta doble dimensión del fenómeno religioso en la actualidad -individualismo y objetividad - será analizada por Taylor en su obra Varie-

[4] "What it Means to Be Secular," [entrevista con Bruce Ellis Benson] Books and Culture, Vol. 8, No 4, (July/August 2002).

[5] Ibíd. 
ties of religión today. ${ }^{6}$ En esta obra, realizada a partir de unas conferencias impartidas por Taylor en Viena en el año 2000, se discuten algunas de las ideas sostenidas por William James acerca de la religión en su famosa conferencia Las variedades de la experiencia religiosa. En opinión de James, la religión se basa en una experiencia individual intensa surgida a través del sentimiento. Cuando se pierden estas señas de identidad, la religión se transforma en un acontecimiento anodino y monótono dando lugar a la aparición de las iglesias o de las costumbres institucionalizadas.

El modo que tiene James de concebir la religión enlaza con una transformación que ha ido produciéndose desde la Edad Media. Fue entonces cuando comenzó a acentuarse un modo de religión basada en el compromiso personal. Este giro se desarrolla posteriormente con el movimiento reformador - alcanzando un lugar destacado la fe frente a las prácticas externas-y continúa, más adelante, con la aparición de los movimientos de devoción propios de la contrarreforma. La concepción que sostiene James de la religión se relaciona con este giro hacia el interior y hacia lo individual, dentro de la línea que defiende una superioridad de los sentimientos frente a la razón.

En opinión de Taylor, esta perspectiva presenta algunas deficiencias. Por una parte, no es capaz de comprender la riqueza de la vida eclesial. Ésta no puede ser definida como una distorsión de una experiencia original, puesto que, en muchas ocasiones, representa el ámbito imprescindible para la aparición y desarrollo de los más variados fenómenos religiosos. La vida religiosa colectiva desempeña un papel primordial, un papel constituyente que no puede reducirse a una mera suma de experiencias individuales. Por otro lado, este énfasis en la experiencia individual, misteriosa e inefable provoca una anulación de cualquier planteamiento teológico. Sin embargo, esta situación no puede sostenerse con firmeza: cualquier experiencia requiere algún modo de formulación, algún tipo de articulación, siendo la articulación teológica la característica del ámbito religioso.

Pero, ante todo, el planteamiento expuesto por James se muestra inapropiado para comprender los dos rasgos que definen, en opinión de Taylor, la vida religiosa actual: la secularización de la esfera pública y el nuevo individualismo. El primero de estos rasgos se desarrolla a partir del universo encantado, sagrado y jerarquizado característico de la Edad Media. Desde la perspectiva medieval, la sociedad reflejaba un orden establecido e inmóvil, en cuya cúspide se encontraba la jerarquía política,

[6] Ch. TAYLOR, Varieties of religión today. William James revisited, Harvard University Press, Cambridge, Massachusetts, 2002. 
situada en un plano superior y sagrado. A lo largo de la Modernidad, este modelo va siendo reemplazado por una cosmovisión en la que la idea de designio adquiere un lugar relevante. Frente a una visión estática acerca del cosmos, nos encontramos ahora ante la necesidad de trabajar para que los fines del universo lleguen a cumplirse, para que el cosmos pueda desarrollar el plan de Dios. Los seres humanos habrán de laborar incesantemente para conseguir que se logre el bienestar querido por el Creador.

Esta nueva perspectiva se desarrollará de modos diferentes en el ámbito anglosajón y en el católico. En el primer caso - y, especialmente en Norteamérica- se ha originado lo que Taylor denomina modo "neo-Durkheimiano": la secularización no provoca, en este ámbito, una separación radical entre la política y la religión; por el contrario, una serie de iglesias o "denominaciones" constituirán diferentes opciones religiosas que conviven junto a otras denominaciones, hallándose en todas ellas presente el sentimiento de pertenecer por igual a una Iglesia mayor que se identifica con una entidad política.

Por el contrario, en el ámbito católico el proceso de secularización ha experimentado un recorrido diferente. El llamado modo "paleo-Durkheimiano" ha tenido una mayor pervivencia. De hecho, en la época barroca, el monarca aún era contemplado como un poder sagrado. Tal vez, por este motivo, la ruptura de este modelo político ha requerido unas transformaciones más violentas y ha generado una comprensión de la vida pública en la que las creencias religiosas han perdido su poder.

El segundo rasgo que descubre Taylor en relación con la religiosidad actual se refiere a la aparición de un nuevo individualismo. Desde la época romántica se ha ido generando una comprensión del sujeto en la que destacan su individualidad y su capacidad para crear la propia vida como si fuera un artista. A partir de la Segunda Guerra Mundial, el individualismo creativo comienza a extenderse, empieza a generalizarse. El nuevo individualismo consiste en la popularización del ideal de autenticidad que reclamaban los románticos.

Cada persona trata de desarrollar su propia vida de un modo original, pero siempre tiene que moverse, tiene que realizar su labor creativa dentro de un espacio simbólico compartido. Nos encontramos, de este modo, ante un individualismo expresivo. Cada sujeto experimenta un momento de soledad — su deseo de ser original y diferente-, pero esta aspiración sólo puede realizarla en un mundo compartido de creaciones.

[7] Taylor emplea estas expresiones recordando el estudio sobre la religión que realiza Durkheim en su obra Las formas elementales de la vida religiosa. 
Este individualismo expresivo provoca un modo de religiosidad personal y original, que se distancia del sentimiento de lealtad hacia una entidad política superior. Se genera, de este modo, una gama de actitudes diferentes: en primer lugar, el aumento de aquellos que se declaran agnósticos o ateos; por otra parte, la aparición de posiciones intermedias: los que abandonan la práctica de la religión pero continúan considerándose miembros de alguna confesión o los que se alejan de la ortodoxia pero creen en algún tipo de fuerza impersonal; en tercer lugar, el incremento de prácticas religiosas no cristianas, principalmente orientales y, por último, el surgimiento de diversas actitudes religiosas que reaccionan contra todo lo anterior redefiniendo la fe de maneras diversas.

En este punto, parece que el planteamiento de William James acerca de la religión se halla en el camino correcto. Sin embargo, su análisis sólo puede reflejar un aspecto de la religión actual, siendo incapaz de ofrecer una explicación adecuada a otros fenómenos importantes: la presencia de numerosos grupos de personas que encuentran en las iglesias el lugar apropiado para su compromiso personal - tales iglesias dejan de identificarse con una entidad nacional, pero representan un modo de enlace colectivo-; la permanencia de identidades nacionales que miran a la religión para reforzar su sentimiento y, por último, la necesidad que sienten muchas personas de experimentar su religión no sólo a través del sentimiento, sino también mediante una disciplina basada en la oración y en la meditación.

\section{Dios en un universo refractado}

Partiendo del análisis que realiza Taylor en torno al fenómeno religioso, podemos extraer algunas conclusiones. En primer lugar, Taylor deja claro que la Modernidad no ha de ser entendida como ausencia de religión. Por el contrario, el proceso de secularización — tal como él lo entiendepone de manifiesto la convivencia entre los principales valores de la Modernidad y el fenómeno religioso. En este sentido, la presencia de la religión sigue siendo un factor muy influyente en las discusiones públicas y en la identificación nacional de algunas comunidades. El modelo de convivencia norteamericano entre la religión y la política es destacado con frecuencia por el filósofo canadiense, quien insiste — siguiendo las tesis de Tocqueville ${ }^{8}$

[8] Cfr. A. Tocqueville, La democracia en América, Fondo de Cultura Económica, México, 1994. 
- en la gran importancia que ha desempeñado la religión en la formación del espíritu cívico de los norteamericanos. ${ }^{9}$

Un segundo punto que merece destacarse es el papel que juega en el terreno religioso la aparición de una nueva forma de individualismo. Es cierto, como indica James, que una cierta interiorización ha caracterizado a la religión desde la Modernidad, pero esta subjetividad no explicaría por sí sola una amplia gama de comportamientos a los que alude Taylor. Por tanto, la presencia de la religión en la actualidad no puede explicarse sólo aludiendo a la subjetivización o interiorización de la experiencia religiosa. La religión requiere algo más: es necesario un trasfondo compartido de prácticas y creencias desde el cual los fenómenos religiosos cobran sentido, es decir, un espacio simbólico común en relación a lo sagrado. Además, ese trasfondo compartido no puede concebirse como una mera suma de experiencias individuales, sino como un trasfondo original y necesario. En este punto Taylor defiende un mayor potencial de la religión católica ${ }^{10}$, dado que la necesidad de un trasfondo compartido permite explicar la importancia de una iglesia organizada o del papel de los mediadores frente a un compromiso únicamente individual.

Pero este segundo rasgo de la religiosidad nos aproxima a una nueva característica: la relación entre la religión y el lenguaje. Para Taylor, la experiencia religiosa es también una experiencia lingüística. En realidad se trata de una experiencia que sigue el modelo epifánico ${ }^{11}$ propio de los autores modernistas. En las creaciones de estos autores, el artista, al mismo tiempo que crea y transita a través de los distintos lenguajes simbólicos, permite la aparición de una realidad que tiene poder propio, que le trasciende. La religión se comporta como el arte epifánico. Se trata, por una parte, de una experiencia individual y personal, pero, por otra parte, sólo es posible realizarla a través de un lenguaje de creencias compartidas. Caminar a través de esos lenguajes significa aproximarse a Dios — pues Dios aparece en ellos-, pero significa también que Dios se manifiesta a

[9] A Taylor le parece muy interesante un concepto de Robert Bellah: la religión civil en América, que aparece en el artículo R. BeLlah, "Civil Religion in America" en Beyond Belief: Essays on Religion in a Post-Tradicional World, Harper \& Row, New York, cap. 9. En este ensayo, América es comparada con Israel, el pueblo elegido. Cfr. Ch. TAYLor, "Religion Today", Transit, 19, (2000).

[10] Cfr. Ch. TAYlon, Varieties of religión today. William James revisited, op. cit., pp. 24-25.

[11] Taylor señala que la obra de arte puede ser el lugar de una epifanía, porque la realidad se revela en el mismo momento de manifestarse la obra. Sin el poder de la obra de arte aquella realidad no podría existir. Como veremos más adelante, Taylor emplea el término epifanía siguiendo la línea emprendida por Joyce. 
través de estos lenguajes con un poder propio y trascendente que lo sitúa más allá del sujeto.

Lo peculiar del mensaje religioso, piensa Taylor, consiste en esta relación recíproca de amor que permite afirmar tanto el ser de Dios como la esencia de la criatura. Esta vinculación es característica del mensaje judeocristiano - idea defendida por Taylor en Fuentes del yo-, pero también - y aquí nos encontramos con un nuevo rasgo del fenómeno religioso- puede encontrarse una relación similar en el budismo.

En el artículo "Spirituallity of life and shadows"12 indica Taylor cómo a lo largo de la historia se han sucedido formas de espiritualidad que establecen diferentes formas de relación entre la apelación a lo trascendente y la reivindicación de la vida. Por un lado, hay formas de religiosidad que emplean la trascendencia simplemente como un instrumento para lograr enriquecer la vida; mientras que, en el extremo contrario, encontramos a los reformadores puritanos defendiendo una auténtica renuncia de la vida. Pero hay, no obstante, una tercera posibilidad: la de aquellos que creen que la apelación a la trascendencia provoca una mayor plenitud de la vida. De este modo, las nociones de ágape del cristianismo o la iluminación de los budistas implican una aspiración a algo que está más allá, pero que conlleva el florecimiento de la vida humana. ${ }^{13}$

Esta sugerencia ofrece un importante punto de contacto entre religiones diferentes. El paralelismo entre el ágape cristiano y la iluminación budista permite romper los límites de una tradición particular y comprender la esencia de la religión de un modo más universal. Este modo de entender la religión puede conducir a comprender la misma no tanto como un conjunto concreto de doctrinas particulares, sino más bien como un modo o una forma de situarse en el mundo. En este sentido, la religión reflejaría una de las facetas características de la identidad contemporánea: junto al racionalismo o al expresivismo, el sentido de la trascendencia jugaría un papel constitutivo del individuo contemporáneo.

En conclusión, podemos señalar, siguiendo a Taylor, que la verdadera experiencia religiosa, conduce siempre a algo más allá, a algo trascendente, sin negar la individualidad. A través de esta experiencia epifá-

[12] "Spirituallity of Life and its Shadow", Compass, 14 (Mayo/Junio), 1996.

[13] Taylor se expresa en una línea similar cuando en su respuesta a Michael L. Morgan indica que podemos comprender y admirar otras formas de espiritualidad diferentes. En este sentido, subraya la tarea de síntesis llevada a cabo por importantes figuras espirituales del siglo XX: Gandhi, Thomas Merton o John Main. Cfr. Ch. TAYlon, "Taylor replies" en J. Tully y D. M. Weinstock (eds.), Philosophy in an age of pluralism, The philosophy of Charles Taylor in question, Cambridge University Press, Cambridge, 1994, p. 229. 
nica, Dios aparece en todo su poder como algo trascendente, pero, al mismo tiempo, el sujeto no queda anulado, puesto que su experiencia se ha producido de un modo personal: Dios resuena a través de un lenguaje personal.

No es de extrañar, por tanto, que encontremos en Taylor una vinculación entre lenguaje y trascendencia. Si vivimos en un mundo refractado, un mundo al que no accedemos directamente sino a través de los lenguajes que configuran nuestro trasfondo cultural, también a Dios se accederá de un modo refractado. Dios forma parte de nuestras tradiciones, de nuestros lenguajes y es a través de ellos cómo encontramos una justificación para su poder. La teodicea de Taylor se adapta al modo contemporáneo de comprender la filosofía, su teoría se sitúa dentro de una perspectiva lingüística. Si Santo Tomás empleaba sus famosas vías para demostrar de modo objetivo la existencia de Dios, si Descartes concebía que la existencia de Dios garantizaba que el sujeto no podía equivocarse en sus razonamientos, Taylor, al mostrar la superioridad de ciertos lenguajes sobre otros, parece descubrir la existencia de Dios en el trasfondo lingüístico-cultural que nos configura. ${ }^{14}$

[14] Una idea similar acerca de la vinculación entre religión y lenguaje es defendida por Anne Fortin - Cfr.: A. Fontin, "Identités religieuses et changement de paradgme. L'imposible historicisme religieux au fondement de la théorie morale de Charles Taylor", en G. Laforest y P. De Lara, (eds.), Charles Taylor et l'interprétation de l'identité moderne, Les presses de L' Université Laval, Quebéc, 1998- y por Michael L. Morgan "Religion, history and moral discourse", en J. Tully. y D. M. Weinstock (eds.), Philosophy in an age of pluralism, op. cit., pp. 49-66. 
